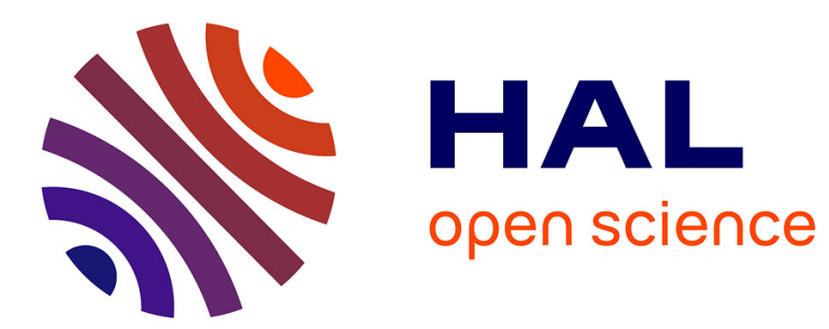

\title{
La théorie des rapports chez Augustus de Morgan
}

Sébastien Gandon

\section{To cite this version:}

Sébastien Gandon. La théorie des rapports chez Augustus de Morgan. 2008. halshs-00296640

\section{HAL Id: halshs-00296640 \\ https://shs.hal.science/halshs-00296640}

Preprint submitted on 14 Jul 2008

HAL is a multi-disciplinary open access archive for the deposit and dissemination of scientific research documents, whether they are published or not. The documents may come from teaching and research institutions in France or abroad, or from public or private research centers.
L'archive ouverte pluridisciplinaire HAL, est destinée au dépôt et à la diffusion de documents scientifiques de niveau recherche, publiés ou non, émanant des établissements d'enseignement et de recherche français ou étrangers, des laboratoires publics ou privés. 


\title{
La théorie des rapports chez Augustus de Morgan.
}

\author{
Sébastien GANDON ${ }^{1}$
}

Résumé : Dans cet article, je présente l'interprétation que A. de Morgan développe de la théorie des rapports euclidiens. Je montre comment la lecture que le mathématicien anglais fait du livre $\mathrm{V}$ des Eléments s'intègre dans le cadre de sa propre pratique mathématique. Je mets également en relation son interprétation à sa nouvelle logique des relations.

Abstract: This article aims at presenting De Morgan's interpretation of the Euclidean theory of ratios. I show that de Morgan's reading of the Elements, Book V, fits in the frame of his own mathematical practice. I also relate De Morgan's interpretation to his new logic of relations.

Pourquoi s'intéresser aujourd'hui à The Connection of Numbers and Magnitude : an Attempt to Explain the Fifth Book of Euclid², court traité consacré à la théorie euclidienne des

\footnotetext{
${ }^{1}$ PHIER, Département de philosophie, Université B. Pascal, Gergovia, 63000 Clermont Université, email : sgandon@orange.fr.
} 
rapports, écrit en 1836 par le mathématicien et logicien anglais Augustus de Morgan ? L'ouvrage n'est manifestement pas le plus important écrit par l'auteur : il n'a été que très peu commenté par les contemporains, et n'a donné lieu à ce jour à aucune étude critique. Son objet d'étude, le livre V des Eléments, se justifie apparemment seulement par l'architecture du cursus universitaire de l'Angleterre du milieu du $\mathrm{XIX}^{\text {ème }}$ siècle $^{3}$; le propos ne semble donc ni mathématiquement, ni épistémologiquement fondamental. Pourquoi donc revenir sur un ouvrage $a$ priori aussi peu attractif?

Notons, tout d'abord, que le travail de A. de Morgan n'a pas été complètement oublié. Thomas Little Heath ${ }^{4}$ comme Bernard Vitrac ${ }^{5}$ mentionnent The Connection of Numbers and Magnitude dans leurs éditions commentées du livre $\mathrm{V}$ des Eléments d'Euclide. B. Vitrac considère la doctrine des «échelles relatives» proposée par de Morgan comme une illustration didactique très efficace de la définition V, 5. T. L. Heath la mobilise constamment

${ }^{2}$ Augustus de Morgan, The connection of numbers and magnitude: an attempt to explain the fifth book of Euclid (Londres, Taylor and Walton, 1836). Cité d'après la réimp. Whitefish, Kessinger Publishing, 2004.

${ }^{3}$ Rappelons que les six premiers livres des Eléments faisaient partie, dans l'Angleterre du début du $\mathrm{XIX}^{\text {ème }}$ siècle, du curriculum de base de la plupart des étudiants en mathématiques (sur l'histoire des programmes universitaire en mathématique dans l'Angleterre du XIXème, voir Joan Richards, Mathematical Visions : The Pursuits of Geometry in Victorian England (Londres, Academic Press, 1988).

4 Thomas Little Heath éd., The Thirteen Books of Euclid's Elements, volume I-III (Cambridge, CUP, 1925). Voir notamment vol. II, 121-124.

${ }^{5}$ Bernard Vitrac éd., Les Éléments d'Euclide, volume I-IV (Paris, PUF, 1990-2001). Voir notamment vol. II, 45-46. 
dans son commentaire. Qu'est-ce exactement que cette théorie des « échelles relatives », et comment s'insère-t-elle dans le contexte plus vaste des recherches de A. de Morgan?

En second lieu, la lecture de The Connection ... révèle que, si le traité est présenté par l'auteur lui-même comme un travail didactique visant à introduire ses étudiants ${ }^{6}$ au livre V des Eléments, il est, en réalité, bien plus que cela. Comme je le montrerai, A. de Morgan tisse des liens extrêmement étroits entre les conceptions d'Euclide et les méthodes mathématiques alors en vogue, qu'il reprend lui-même dans ses travaux plus pointus. La théorie des rapports eudoxiens est ainsi considérée comme exemplifiant des principes qui sont ceux-là mêmes qui guident le savant anglais dans ses recherches algébriques.

Nous verrons notamment (section 1) que A. de Morgan voit, dans le livre V, la mise en place d'une pure forme, à laquelle manquerait une interprétation. Les « échelles relatives », fourniraient ainsi une représentation intuitive à une notion (celle de rapport) dont seules les règles d'usage sont données, exactement comme dans un tout autre contexte (alors au centre des préoccupations mathématiques), la représentation géométrique des nombres imaginaires permet d'interpréter les règles d'addition et de multiplication entre complexes.

Nous étudierons également (section 2) comment la théorie des rapports de rapport est reliée au calcul des opérations, que A. de Morgan, parmi d'autres (Duncan H. Gregory, G. Peacocke...) formant ce qu'il est convenu d'appeler l'«école algébrique anglaise », développe à la même époque. Penser le rapport lui-même comme une grandeur exige le même cheminement intellectuel que celui nécessaire pour concevoir une opération sur des quantités comme étant elle-même une quantité.

Enfin, nous verrons comment (section 3), dans des textes plus tardifs, A. de Morgan établit un lien conceptuel entre cette doctrine de la composition des rapports et sa théorie relationnelle du syllogisme. La « loi suprême» du syllogisme se donne alors comme une

\footnotetext{
${ }^{6}$ A. de Morgan fut professeur à l'University College de Londres de 1828 jusqu'à 1866.
} 
forme généralisée de l'opération de composition des rapports : « une relation de $X$ à $Y$ composées d'une relation de $Y$ à $Z$ donne une relation de $X$ à $Z$ ».

Loin d'être un exercice purement didactique, isolé du reste de l'œuvre, la lecture que A. de Morgan propose d'Euclide renvoie ainsi de façon serrée aux préoccupations et thématiques centrales de l'auteur. Le but de cet article est d'abord de rendre manifeste cette interconnexion ${ }^{7}$.

Mais un autre motif, plus sujet à controverse et plus indirect, m'a poussé à revisiter ces textes oubliés. La doctrine relationnelle du syllogisme est une des approches dont Russell s'inspire lorsqu'il rompt avec la théorie peanienne et met en place sa nouvelle logique ${ }^{8}$. Or la logique des relations, sous la forme de la logique du premier ordre (polyadique), a été utilisée par Jean-Louis Gardiès pour formuler une interprétation du livre V des Eléments ${ }^{9}$. Une telle lecture, d'inspiration logique, a suscitée de nombreuses critiques : important des concepts et des outils complètement étrangers au texte euclidien, Gardiès gauchirait, bien plus qu'il n'éclairerait, le sens des textes ${ }^{10}$. La question soulevée est la suivante : peut-on et doit-on,

\footnotetext{
${ }^{7}$ Il ne s'agit donc pas ici d'évaluer la singularité de la lecture proposée par A. de Morgan par rapport à l'histoire des interprétations du livre $\mathrm{V}$, ni non plus d'en évaluer la vérité ou la pertinence.

${ }^{8}$ Voir Bertrand Russell, The Principles of Mathematics, (Londres, Routledge, 1903), 23-26, 218-226

${ }^{9}$ Voir notamment Jean-Louis Gardiès, L'héritage épistémologique d'Eudoxe de Cnide, (Paris, Vrin, 1988), qui va jusqu'à fonder une distinction entre des corpus (l'un étant attribué à Eudoxe, l'autre à Thééthète) à l'intérieur des Éléments sur des distinctions logiques.

${ }^{10}$ Voir notamment la discussion de B. Vitrac dans Les Éléments d'Euclide, volume II (Paris, PUF, 1994), 552-554.
} 
sous prétexte qu'ils seraient universels, user des concepts et des distinctions logiques pour interpréter les textes mathématiques du passé ?

Je laisserai ici ce problème de côté. Dans ce qui suit, je souhaite simplement suggérer la chose suivante. Il est possible qu'il y ait eu, historiquement parlant, un lien étroit entre l'élaboration de la théorie logique des relations dans la seconde moitié du XIX ${ }^{\text {ème }}$ siècle et la lecture du livre V des Eléments. Dans cette hypothèse, l'usage de la logique comme outil heuristique dans le déchiffrage de la théorie des rapports ne constituerait pas une application anhistorique d'une machinerie sortie toute armée de la tête de Russell : la genèse de la nouvelle logique aurait, dès le début, partie liée à une méditation et à une lecture du texte euclidien. Ce résultat, s'il s'avérait fondé, ne trancherait pas sur le fond le débat ouvert par les travaux de Gardiès. En esquissant une histoire de l'interprétation relationelle de la théorie des rapports, notre étude pourrait néanmoins contribuer à mettre la discussion en perspective.

\section{I- La théorie des échelles relatives.}

Résumons la construction présentée dans les vingt premières pages de The Connection of Numbers and Magnitudes. Après avoir défini les grandeurs d'un même genre comme étant des entités capables d'être additionnées et ordonnées, de Morgan définit la notion d'échelle des multiples de $A$. « The scale of multiples of $A$ » est simplement l'ensemble des multiples de $A: A, 2 A, 3 A, \ldots$ L'auteur complète alors sa définition de la grandeur par un équivalent de l'axiome d'Archimède, qu'il formule ainsi : « aussi petit que $A$ puisse être, ou aussi grand que $B$ puisse être, les multiples dans l'échelle $A, 2 A, \ldots$ seront à un moment en position de surpasser $B$, si on continue l'échelle suffisamment loin : $B$ et $A$ étant des grandeurs de même 
genre ${ }^{11}{ }^{1}$. De ceci, A. de Morgan déduit en premier lieu, par l'absurde, le principe d'exhaustion - c'est-à-dire le fait que si d'une grandeur la plus grande partie est ôtée, et si du reste la plus grande partie est ôtée, et ainsi de suite, alors le reste peut devenir aussi petit que l'on veut ; puis, il dérive le théorème suivant, présenté comme central :

Que soient données deux grandeurs du même genre, $A$ et $B$, et que soient formées les échelles des multiples $A, 2 A, \ldots B, 2 B, \ldots$ alors une de ces deux choses doit être vrai ; OU BIEN il y a des multiples dans la première échelle qui sont égaux à ceux de la seconde échelle ; OU il y a des multiples dans la première échelle qui sont aussi près que nous voulons des multiples (peut-être pas les mêmes) dans le second ensemble. ${ }^{12}$

La démonstration de ce qui revient à prouver la densité de $\mathrm{Q}$ ?? dans $\mathrm{R}$ ?? s'effectue par le principe d'exhaustion. Deux grandeurs qui ont certains de leurs multiples en commun sont appelées commensurables; deux grandeurs qui n'ont aucun de leurs multiples en commun sont appelées incommensurables.

De Morgan définit alors son concept clé, celui d'échelle relative :

Nous pouvons (...) former ce que nous pouvons appeler une échelle relative de multiples en notant les multiples de $A$, et en y insérant les multiples de $B$ à leurs places propres ; ou vice versa. ${ }^{13}$

\footnotetext{
${ }^{11}$ De Morgan, The Connexion..., op. cit. in note 2, 4.

${ }^{12}$ Ibid., 6

${ }^{13}$ Ibid., 20.
} 
L'échelle relative de $A$ et de $B$ manifeste la répartition des multiples de $B$ au sein de l'échelle de multiples de $A$, et c'est cette échelle que A. de Morgan identifie au rapport eudoxien. Comment justifie-t-il cette identification?

Après avoir présenté son concept d'échelle relative, de Morgan se focalise sur ce que l'on a coutume d'appeler l'algorithme d'Euclide. Il rappelle d'abord que rien n'oblige à limiter le champ d'application de cet algorithme aux nombres (rien d'original $\mathrm{ici}^{14}$ ) ; puis il montre que son application à deux grandeurs quelconques (qu'elles soient commensurables ou non) équivaut à construire l'échelle relative des deux grandeurs. Le point étant méthodologiquement important, expliquons plus en détail comment de Morgan procède.

Le mathématicien, sans le nommer, reprend en réalité la théorie classique de Lagrange sur les fractions continues ${ }^{15}$ : il donne la relation de récurrence déterminant la valeur de la réduite d'ordre $n, u_{n}=\frac{p_{n}}{q_{n}}$, c-à-d la valeur de la fraction approchant le rapport des deux grandeurs $A$ et $B$ au bout de $n$ étapes de l'algorithme d'Euclide. Si $A=a_{0} B+r_{1}$ avec $r_{1}<B$ et si $B=a_{1} r_{1}+r_{2}$ avec $r_{2}<r_{1}$, alors :

$$
\begin{aligned}
& p_{n}=a_{n} p_{n-1}+p_{n-2} \text { avec } p_{0}=a_{0} \text { et } p_{1}=a_{0} a_{1}+1 \\
& q_{n}=a_{n} q_{n-1}+q_{n-2} \text { avec } q_{0}=1 \text { et } q_{1}=a_{1} .
\end{aligned}
$$

\footnotetext{
${ }^{14}$ Ibid., 24 .
}

${ }^{15}$ Lagrange, J.-L., Sur la résolution des équations numériques, Mémoires de l'Académie de Berlin, 23, 1769, reimpr. in Euvres Complètes, (Paris, Gauthiers-Villars, t. II, 1868), 539578. 
On sait, d'après Lagrange, que les fractions réduites sont irréductibles -- que la suite des réduites de rang pair est croissante, que celle de rang impair est décroissante, et que les deux suites tendent toutes vers le nombre réel $R$ mesurant le rapport de $A$ à $B$ :

$$
\begin{aligned}
& u_{2 n}<u_{2 n+2}<R<u_{2 n+1}<u_{2 n-1} \\
& \quad \text { avec }\left|u_{n+1}-u_{n}\right| \text { tend vers } 0 \text { quand } n \text { tend vers l'infini. }
\end{aligned}
$$

A. de Morgan voit dans ces résultats l'ensemble des conditions permettant la formation, à partir de l'algorithme d'Euclide, d'une échelle relative des multiples de deux grandeurs quelconques : des relations entre les réduites, explique-t-il, « nous pouvons déterminer si n'importe quel multiple donné de $A, x A$, est plus grand ou plus petit que n'importe quel multiple de $B, y B »$, ou encore « entre quels multiples consécutifs de $B$ se trouve $x A{ }^{16}$. En effet, il s'agit de savoir si $x A>y B$ ou si $x A<y B$, c'est-à-dire si $A>$ ou $<\frac{y}{x} B$. Si $A$ et $B$ sont commensurables, alors par définition il existe deux entiers $p$ et $q$ tels que $p A=q B$, et la comparaison de $\frac{q}{p}$ et de $\frac{y}{x}$ tranche la question. Si $A$ et $B$ sont incommensurables, alors quels que soient $p$ et $q$ entiers, on a $A=\frac{q}{p} B+K$, avec $K$ différent de zéro. Donc ici, puisque $x$ et $y$ sont donnés, on a $A=\frac{y}{x} B+K$, avec $K$ différent de zéro -- or comme, d'après (1), la différence entre $u_{2 n+1}$ et $u_{2 n}$ des réduites du développement d'ordre $2 n$ et $2 n+1$ en fraction continue de $A$ par $B$ peut être rendue aussi petite que l'on veut, que la suite des réduites de rang pair croît, que la suite des réduites de rang impair décroît, il existe nécessairement un

\footnotetext{
${ }^{16}$ De Morgan, The Connexion..., op. cit. in note 2, 15.
} 
rang $n^{\prime}$, tel que $u_{2 n^{\prime}}<\frac{y}{x}<u_{2 n^{\prime}+2}$ ou $u_{2 n^{\prime}+1}<\frac{y}{x}<u_{2 n^{\prime}-1}$. Dans le premier cas, $x A>y B$, et dans le second $x A<y B$.

Prenons l'exemple du nombre d'or $\left(R=\frac{1+\sqrt{5}}{2}\right)$ développé par de $\operatorname{Morgan}^{17}: a_{0}=1$ et $a_{1}=1$, et où donc :

$$
\frac{1+\sqrt{5}}{2}=\frac{1}{1+\frac{1}{1+\frac{1}{1+\ldots}}}
$$

On a (les $p$ et les $q$ désignant les numérateurs et dénominateurs des réduites) :

\begin{tabular}{|l|l|l|l|l|l|l|l|l|l|l|l|l|l|}
\hline rang & 0 & 1 & 2 & 3 & 4 & 5 & 6 & 7 & 8 & 9 & 10 & 11 & etc \\
\hline$p$ & 1 & 2 & 3 & 5 & 8 & 13 & 21 & 34 & 55 & 89 & 144 & 233 & etc \\
\hline$q$ & 1 & 1 & 2 & 3 & 5 & 8 & 13 & 21 & 34 & 55 & 89 & 144 & etc \\
\hline
\end{tabular}

De ce tableau, A. de Morgan tire que :

$$
A>B<2 B>\frac{3}{2} B<\frac{5}{3} B>\frac{8}{5} B<\frac{13}{8} B \ldots
$$

c'est-à-dire que :

${ }^{17}$ Ibid., 19. 
$A$ est entre $B$ et $2 B$

$2 A$ est entre $3 B$ et $4 B$

$3 A$ est entre $4 B$ et $5 B$

$4 A$ est entre $6 B$ et $7 B$

$5 A$ est entre $8 B$ et $9 B$

etc.

Ou encore : $B, A, 2 B, 3 B, 2 A, 4 B, 3 A, 5 B, 6 B, 4 A, 7 B, 8 B, 5 A, 9 B$, etc...

Les réduites d'ordre $n$ du développement en fraction continue des deux grandeurs permettent donc de construire, que les grandeurs soient ou non commensurables, une échelle relative des multiples. Deux grandeurs étant données, grâce à l'algorithme d'Euclide, l'échelle relative des multiples est donnée. A. de Morgan note immédiatement que, bien évidemment, la converse n'est pas vraie : si l'échelle relative est donnée, les deux grandeurs définissant le rapport ne sont pas nécessairement données. «Qu'est-ce donc », demande-t-il alors, « qui est donné, lorsque l'échelle est donnée ? ». L'échelle définit une certaine relation qui appartient à $2 A$ et $2 B, 3 A$ et $3 B$, etc $\ldots$ aussi bien qu'à $A$ et $B$, nommée précisément proportion ou rapport :

Par le rapport de $A$ à $B$, nous entendons (sans autre spécification pour l'instant) une relation entre les grandeurs $A$ et $B$, déterminée par la façon dont les multiples de $A$ sont distribués, si chacun est écrit entre les plus proches multiples de $B$ en grandeur. C'està-dire que si $B, 2 B, 3 B, \ldots$ et $A, 2 A, 3 A, \ldots$ sont formés, et si $A$ se trouve entre $B$ et $2 B, 2 A$ entre $2 B$ et $4 B$, et ainsi de suite, l'échelle relative (...) doit être le seul élément 
déterminant du rapport, de sorte qu'il n'y ait rien que l'ordre de cette échelle dont le rapport dépende. ${ }^{18}$

Après avoir montré la proximité entre l'idée commune de proportion et le concept d'échelle relative, de Morgan prouve à partir de sa définition les principaux théorèmes du livre $\mathrm{V}^{19}$.

Mais revenons à l'essentiel, c'est-à-dire à la distinction, dans le raisonnement du mathématicien, de deux moments : celui de la définition de la notion d'échelle relative d'une part, celui de son identification à la notion eudoxienne de rapport de l'autre. Pour saisir la portée d'une telle distinction, il est nécessaire de revenir sur la façon tout à fait particulière dont A. de Morgan caractérise la démarche empruntée par Euclide dans les Eléments. Citons l'auteur :

(Lors de l'exposé de la doctrine des échelles relatives), nous avons déduit la nécessité de considérer les incommensurables comme Euclide les conçoit, d'une notion qui, appliquée aux commensurables, admet une représentation définie, dérivée de l'idée de proportion. Mais la méthode du livre $\mathrm{V}$ est différente. On y fait là l'hypothèse que partout où deux grandeurs existent, leur existence jointe donne lieu à une troisième

\footnotetext{
${ }^{18}$ Ibid. 23.
}

${ }^{19}$ Suivant la voie ouverte par Knorr, D. Fowler (in The Mathematics of Plato's Academy: A New Reconstruction (Oxford, Clarendon Press, 1987)) a développé une interprétation « antyphérétique» des «ratios» euclidiens, dans lequel un usage élaboré de la théorie lagrangienne des fractions continues est mobilisée. S'il semble que A. de Morgan (que Fowler ne cite pas) ait anticipé cette ligne, la définition du rapport qu'il propose n'est pas antyphérétique (voir plus loin). 
grandeur, appelée leur rapport, dont aucune conception n'est donnée, à part certaines directives concernant la façon de leur appliquer les termes égales, plus grandes et plus petites. $^{20}$

En modernisant la terminologie, on pourrait dire que de Morgan attribue à Euclide une approche purement formelle : le mathématicien grec donnerait dans le livre $\mathrm{V}$ les règles d'usage d'un symbole, celui de « rapport », sans pour autant fournir une « conception » à laquelle renvoyé la notion. Dans le cas commensurable, une représentation intuitive du rapport (sous forme de fraction numérique) est, certes, disponible; mais dans le cas incommensurable, aucune représentation déterminée n'est associée au terme défini. En creux se dessine alors une tâche : celle de donner une interprétation, d'élaborer une « conception », satisfaisant les critères simplement formels fournis dans le libre V. C'est précisément à ce travail que dit s'être attelé A. de Morgan. L'algorithme d'Euclide joue dans ce projet un rôle déterminant, quoique assez subtile. Le mathématicien anglais n'utilise pas, comme certains commentateurs médiévaux d'expression arabe (notamment al-Kayyâm) ${ }^{21}$, l'anthyphérèse pour donner directement un contenu à la notion de rapport de deux grandeurs; il s'en sert pour assurer qu'il y a une procédure effective permettant d'engendrer « l'échelle relative » à partir des deux grandeurs. Autrement dit, le rapport n'est pas directement identifié par A. de Morgan à la suite des quotients partiaux obtenus par l'algorithme d'Euclide. Il est associé à l'« échelle relative » des deux grandeurs, l'anthyphérèse fournissant l'algorithme permettant de construire pas à pas ce nouvel objet. Le point crucial pour A. de Morgan est sans doute que

${ }^{20}$ Ibid., p. 62

${ }^{21}$ Sur la théorie des proportions de Al-Khayyâm, voir l'extrait de la Seconde Épitre sur l'Evocation de la Proportion, trad. A. Djebbar, in Evelyne Barbin et alii éds, Histoire d'algorithmes. Du caillou à la puce (Paris, Belin, 1994), 134-138. 
l'échelle relative, à la différence de la suite des quotients partiaux, fournit une représentation intuitive, que l'on peut (partiellement) dessiner, des rapports euclidiens.

L'objectif du mathématicien serait bien, en un sens, pédagogique : si rien n'est associé au mot rapport, il ne faut pas s'étonner que les étudiants ne voient pas de quoi il s'agit. Mais il ne se réduit pas à cela. A. de Morgan présente, dans On the Foundation of Algebra $(1842)^{22}$, une distinction entre technical algebra (un ensemble de symboles et de loi de combinaison sans signification) et logical algebra (un calcul interprété, dirions-nous aujourd'hui). Citons un passage justement célèbre du premier chapitre du second livre de son traité Trigonometry and Double Algebra :

${ }^{22}$ A. de Morgan, On the Foundations of Algebra, Trans. Camb. Proc. Soc., VII (1842), 173187: «L'Algèbre consiste (...) en deux parties, la technique, et la logique. L'algèbre technique est l'art d'employer les symboles en suivant des règles, qui, lorsque cette partie du sujet est considérée indépendamment de l'autre, sont prescrites comme étant les définitions des symboles. L'algèbre logique est la science qui examine la méthode consistant à donner une signification aux premiers symboles, et à interpréter tous les résultats symboliques qui en suivent. » Sur cette distinction centrale, voir Marie-José Durand-Richard, La genèse de l'algèbre symbolique : une influence possible de J. Locke, Revue d'histoire des sciences, 43 (1990), 129-180, et Joan Richards, A. de Morgan, The History of Mathematics and the Foundations of Algebra, Isis, 78 (1987), 6-30.

Notons que la conception « formaliste » des mathématiques, sous-jacente à la distinction entre algèbre technique et algèbre logique, est adoptée dès 1835 (un an avant la publication de The Connection...), lors de la revue que A. de Morgan consacre à Treatrise on Algebra de Peacocke. Sur l'évolution de A. de Morgan, voir Helena M. Pycior, A. de Morgan's Algebraic Work : The Three Stages, Isis, 74/2 (1983), 211-226. 
Dès que l'idée de maîtriser des symboles et des lois de combinaison, dépourvus de signification, est devenue familière, l'étudiant a la notion de ce que j'appellerai un calcul symbolique : un art, non une science ; et selon toute apparence un art inutile, sauf en ce qu'il peut après coup fournir la grammaire d'une science. (...) Supposons l'étudiant sans possibilité d'obtenir (la) signification (du calcul symbolique) de l'extérieur: son professeur est mort, et il doit inventer des signification pour luimême. Son problème est : étant donné des symboles et des lois de combinaison, donner aux symboles des significations telles que le droit de les combiner de cette manière suivent comme une conséquence logique. Il essaie et réussit (...). Il a transformé le calcul symbolique en un calcul pourvu de sens. ${ }^{23}$

La situation pédagogique est présente ici, mais avant tout comme métaphore. A de Morgan l'utilise pour décrire un type de recherche mathématique qui vise à fournir une interprétation, un meaning, à un calcul symbolique déjà existant. La situation de l'étudiant dont le maître est mort est précisément celle de A. de Morgan lui-même - c'est-à-dire, dans l'ouvrage dont le passage est extrait, celle du mathématicien cherchant à interpréter les calculs sur les nombres complexes $^{24}$

${ }^{23}$ Augustus de Morgan, Trigonometry and double algebra (Londres, Taylor, Walton and Maberly, 1849), 92-93.

${ }^{24}$ Commentant le nom « double algebra », de Morgan conclut sa préface ainsi: « Si, grâce à l'application d'un adjectif quelque peu surprenant au mot algèbre, n'importe lequel de ceux qui sont encore déroutés par un art dans lequel des quantités impossibles, ou des quantités qui ne sont pas des quantités, sont prises pour objet d'étude, devait réaliser que, de façon insensible et grâce au travail de nombreux esprits, l'art est devenu une science et les 
Replacé dans ce contexte, le texte de The Connection... cité plus haut prend tout son sens: l'exposition euclidienne y est présentée comme la mise en place d'une algèbre technique, conduite directement en grec $^{25}$, le but de A. de Morgan étant de transformer ce calcul symbolique en calcul pourvu de sens, de donner à ce qui n'est qu'une forme vide un meaning. La démarche de A. de Morgan serait ainsi la suivante : la notion de grandeur relative a un sens, une interprétation évidente lorsque les deux grandeurs sont commensurables. Comment étendre l'interprétation aux autres cas? Seul l'algorithme d'Euclide permet de répondre à cette demande, car il fournit une procédure effective pour construire l'échelle relative des deux grandeurs. A. de Morgan, grâce à sa construction, étendrait l'interprétation de la notion de rapport aux cas incommensurables, tournerait ce qui est conçu comme la mise en place d'un calcul simplement formel (la définition $\mathrm{V}$, 5 d'Euclide) en un symbolisme pourvu de sens ${ }^{26}$.

L'approche de A. de Morgan n'est donc pas seulement une formulation élégante et didactiquement efficace de la définition euclidienne; elle est liée aux recherches impossibilités possibles, alors ceux là, tout du moins, ne devrait adresser aucune objection à l'expression. 》

${ }^{25}$ A. de Morgan précise (Trigonometry and double algebra, op. cit. in note 23, 91-92) qu'une algèbre peut très bien ne pas avoir de symboles propres et user des mots du langage courant. ${ }^{26}$ L'interprétation développée par de Morgan connaîtra un certain succès. Outre les éditeurs d'Euclide, Heath en Angleterre, Vitrac en France, des mathématiciens, jusqu'au début du XXème siècle, continueront à se référer à la notion d'échelle relative. Micaiah John Muller Hill, professeur à Cambridge, consacrera ainsi deux ouvrages à la théorie des échelles relatives - voir The content of the fifth and sixth books of Euclid (Cambridge, CUP, 1900) et The theory of proportion (Londres, Constable, 1914). 
mathématiques et aux conceptions épistémologiques de l'auteur. Pour A. de Morgan, $\sqrt{-1}$ comme la notion euclidienne de rapport doivent être dotés d'un «meaning », c'est-à-dire doivent être associés à des représentations «concrètes », dont l'ensemble des règles gouvernant leur emploi peuvent être extraites. Remarquons que, dans le cas euclidien, cette tâche exige que l'on combine des méthodes souvent mises en concurrence dans la littérature secondaire : la définition eudoxienne du rapport et les procédures antyphérétiques. Notons enfin, que l'interprétation proposée par de Morgan n'a pas la prétention d'être historiquement fidèle : le but n'est pas de déterminer ce que Euclide ou Eudoxe cherchait, mais de fournir une interprétation possible, non unique, de la notion de rapport ${ }^{27}$.

Ces différents traits, réception des Eléments à partir des recherches sur les calculs et leurs interprétations, application aux théories euclidiennes de catégories épistémologiques élaborées pour rendre compte des mathématiques qui lui sont contemporaines, caractérisent également un autre aspect, moins célèbre, de l'approche de A. de Morgan vers laquelle nous allons maintenant nous tourner : sa théorie de la composition des rapports.

\section{II- La composition des rapports.}

${ }^{27}$ La suite du passage cité (Trigonometry and double algebra, op. cit. in note $23,92-93$ ) audessus le précise: «(L’élève) a-t-il fourni ce que son professeur lui aurait donné, s’il avait vécu ? En un sens, certainement : il a transformé son calcul symbolique en un calcul pourvu de sens. Mais il ne s'ensuit pas qu'il l'ait fait comme son professeur le lui aurait appris, s'il avait vécu. » La distinction entre jeu de symboles et interprétation est bien entendu complètement étrangère au contexte des mathématiques euclidiennes. Encore une fois, ce n'est pas la pertinence de l'interprétation de A. de Morgan qui nous intéresse ici. 
La composition des rapports nécessite, dit de Morgan, un pas supplémentaire dans l'abstraction. Il s'agit de concevoir les relations entre grandeurs que sont les rapports, comme étant eux-mêmes des grandeurs, c'est-à-dire comme étant eux-mêmes des entités susceptibles de plus et de moins, pouvant être mesurées. Citons un extrait de l'article « Ratio » écrit pour la Penny Cyclopedia :

Le nouveau pas à accomplir est plutôt d'un genre abstrait : le rapport lui-même est une grandeur, ou encore la grandeur relative d'une grandeur par rapport à une autre est elle-même une grandeur. Pour comprendre cela, rappelons que par grandeur, nous ne voulons pas seulement parler de la grandeur physique, comme la taille, le poids, la longueur, etc., mais de n'importe quelle conception mentale telle qu'il est évident que soit plus grand, soit égal, soit plus petit s'applique à deux quelconques d'entre elles, et telle qu'un mode permettant de mesurer l'excès et le défaut (...) puisse être trouvé. ${ }^{28}$

A. de Morgan cherche donc à définir une relation d'ordre entre rapports et une addition des rapports sur le modèle de celui des longueurs. Pour comparer deux grandeurs $A$ et $B$, il faut faire coïncider une de leurs extrémités, et voir quel segment est recouvert par l'autre. De même pour comparer deux rapports, il faut faire coïncider une de leurs « extrémités », c'est-àdire trouver un terme commun aux deux rapports :

Quel est le plus grand rapport, celui de $P$ à $Q$, ou de $A$ à $B$ ? Choisissons une quantité quelconque, $Z$, et posons que le rapport de $A$ à $B$ est celui de $X$ à $Z$, tandis que celui de $P$ à $Q$ est celui de $Y$ à $Z$. La question se réduit ainsi à la suivante : quel est le plus grand, $X$ considéré relativement à $Z$ ou $Y$ relativement au même $Z$ ? La réponse dépend

\footnotetext{
${ }^{28}$ A. de Morgan, Article 'ratio' in The Penny Cyclopedia, 1842, 49-53.
} 
évidemment de savoir celui qui est absolument le plus grand des deux, $X$ ou $Y$. Supposons que $X$ soit le plus grand, alors le rapport de $A$ à $B$ est plus grand que celui de $P$ à $Q{ }^{29}$

D'autre part, pour évaluer l'excès d'une longueur par rapport à une autre, il faut former le segment défini par les deux extrémités des grandeurs $A$ et $B$ lorsqu'elles ont leur autre extrémité en commun; il en va de même pour les rapports : pour évaluer l'excès d'un rapport par rapport à un autre, il faut former le rapport défini par les deux grandeurs-extrémités :

Mais en quelle relation le rapport de $A$ à $B$ est-il plus grand que celui de $P$ à $Q$ ? Répétons la transformation, et le rapport de $X$ à $Z$ est plus grand que le rapport de $Y$ à $Z$, exactement de la même façon que $X$ est plus grand que $Y$-à savoir, le rapport de $X$ à $Y$ est le rapport du rapport de $A$ à $B$ à celui de $P$ à $Q$. Donc, pour trouver le rapport de deux rapports, à savoir $A$ à $B$, et $P$ à $Q$, posons

$$
\begin{aligned}
& A: B:: X: Z \\
& Q: P:: Z: Y
\end{aligned}
$$

et le rapport de $X$ à $Y$ est le rapport cherché. Ce procédé est appelé par Euclide la composition des rapports de $A$ à $B$ et de $Q$ à $P$.

A. de Morgan, dans The Connection ... comme dans l'article « Ratio » de la Penny Cyclopedia, pousse d'ailleurs la comparaison plus loin. De même que l'on peut définir, à partir d'une grandeur quelconque $A$ une échelle de ses multiples, de même il est possible de définir à partir d'un rapport quelconque, une échelle des rapports qui sont en proportion avec lui. Ainsi, si $I$ représente le segment unité et $V$ un segment quelconque plus grand (disons) que 
$I$, et si $\wp$ désigne le rapport $V: I$, alors nous pouvons former l'échelle des rapports $\wp_{1}\left(=V_{1}\right.$ : $V), \wp_{2}\left(=V_{2}: V_{1}\right)$, etc $\ldots$, tels que $\mathrm{e}^{30}$ :

$$
V: I \quad: \because \quad V_{1}: V \quad: \because \quad V_{2}: V_{1}
$$

Il est également possible de représenter les rapports $\wp_{-1}, \wp_{-2}$, etc... tels que :

$$
I: V \quad: \because \quad V_{-1}: I \quad: \because \quad V_{-2}: V_{-1} \quad: \because \quad \ldots
$$

Cette échelle joue pour les rapports le rôle que jouait l'échelle des multiples pour les longueurs. Grâce à elle, si un rapport donné quelconque était pris comme mesure, tous les autres rapports pourraient être mesurés par lui. A de Morgan effectue le lien entre ces échelles de rapports et la façon dont les premiers logarithmes ont été calculés ${ }^{31}$. En conservant notre terminologie, $n$ est le logarithme de base $\wp$ de $\wp_{n}$ (par exemple, si $\wp_{n}=2^{4}=16$, alors $\left.\ln _{2} 16=4\right)$

Selon S. Rommevaux ${ }^{32}$, la comparaison, centrale chez de Morgan, de la composition avec l'additivité est au cœur de la théorie des rapports des rapports, telle qu'elle a été élaborée par Oresme et les médiévaux. En reprenant une démarche alors classique, A. de Morgan ne

${ }^{30}$ Voir A. de Morgan, The Connexion ..., op. cit. in note 2, 73-76.

${ }^{31}$ Ibid. 79-81.

${ }^{32}$ Sabine Rommevaux, Thomas Bradwardine, Traité sur les rapports ; Nicole Oresme, Sur les rapports de rapports, Paris, Les Belles Lettres (2007). Notons que la théorie de la composition des rapports ne se trouve pas explicitement dans les Eléments ; sur ce point, voir Ken Saito, Compounded Ratio in Euclid and Apollonius, Historia Scientiarum 31 (1986), 25 59. 
ferait donc pas ici preuve de grande originalité. Mais si le contenu doctrinal n'est pas nouveau, la manière dont de Morgan l'articule aux recherches algèbriques du XIXème siècle anglais est extrêmement singulière. C'est vers cet aspect que je vais maintenant me tourner.

Un des principes important mis en oeuvre de façon systématique par les membres de « l'école algébrique anglaise » (dont de Morgan fut, avec Duncan H. Gregory, G. Peacocke et G. Boole un des plus illustres représentants) ${ }^{33}$, était le principe dit de « séparation des symboles ", qui consistait à regrouper, dans une équation, les symboles d'opérations, et à traiter le groupe résultant comme un signe usuel de quantité. Plus qu'un long discours, l'exemple suivant permettra de fixer les idées ${ }^{34}$.

On sait que pour tout $x$ réel ou complexe :

$$
e^{x}=1+x+\frac{x^{2}}{2 !}+\ldots+\frac{x^{n}}{n !}+\ldots
$$

Or, si $u$ est une fonction réelle ou complexe infiniment dérivable (et soumis à des conditions supplémentaires de régularité), la formule de Taylor nous dit que :

\footnotetext{
${ }^{33}$ Sur cette « école », voir Marie-José Durand-Richard, Le réseau des algébristes anglais et la symbolisation de l'opératoire $(1812-54), \mathrm{ms}$, http://www.rehseis.cnrs.fr/calculsavant/Equipe/Resources/mjdr_1999.pdf (1999), et Elaine Koppelmann, The calculus of operations and the rise of abstract algebra, Archive for History of Exact Sciences, 8 (1971), 155-242

${ }^{34}$ Il s'agit en réalité de bien plus qu'un exemple: l'analogie entre la formule de Taylor et le développement en série de l'exponentiel, notée déjà par Leibniz, a nourri l'histoire du calcul des opérations. Voir sur ce point E. Koppelman, op. cit. in note 33.
} 


$$
u(x+h)=u(x)+\frac{h}{1 !} \frac{d u}{d x}(x)+\frac{h^{2}}{2 !} \frac{d^{2} u}{d x^{2}}(x)+\ldots+\frac{h^{n}}{n !} \frac{d^{n} u}{d x^{n}}(x)+\ldots
$$

Leibniz, puis Lagrange, sont les premiers à avoir noté la similarité entre (2) et (3) qui permet d'écrire :

$$
e^{h \frac{d u}{d x}}=u(x)+\frac{h}{1 !}\left(\frac{d u}{d x}\right)^{1}(x)+\frac{h^{2}}{2 !}\left(\frac{d u}{d x}\right)^{2}(x)+\ldots+\frac{h^{n}}{n !}\left(\frac{d u}{d x}\right)^{n}(x)+\ldots
$$

à condition de remplacer pour tout $n$ entier $\left(\frac{d u}{d x}\right)^{n}$ par $\frac{d^{n} u}{d x^{n}}$. Il n'y a là, dans un premier temps, rien de plus qu'une élégante convention d'écriture. Mais le mathématicien français Louis François Antoine Arbogast a vu dans ce changement de formulation l'application d'une méthode générale, baptisée «méthode de séparation des échelles d'opération ». Il « sépare », en effet, dans (4), ce qui relève de l'opératoire de ce qui concerne la fonction $u$, et écrit donc l'égalité sous la forme :

$$
e^{h \frac{d u}{d x}} \times u=u(x)+\frac{h}{1 !}\left(\frac{d u}{d x}\right)^{1}(\mathrm{x})+\frac{h^{2}}{2 !}\left(\frac{d u}{d x}\right)^{2}(\mathrm{x})+\ldots+\frac{h^{n}}{n !}\left(\frac{d u}{d x}\right)^{n}(\mathrm{x})+\ldots
$$

En appelant $\mathbf{E}$ l'opérateur qui, appliqué à $u(x)$ donne $u(x+h)$ (c-à-d tel que : $\mathbf{E} u(x)=u(x+h)$ ), Arbogast pose que :

$$
\mathbf{E}=e^{h \frac{d u}{d x}}
$$


Le mathématicien français prend ainsi au pied de la lettre ce qui s'esquissait dans la reformulation de (2) en (3) et, séparant les symboles d'opération des symboles de quantité, définit un nouvel objet, l'opérateur $\mathbf{E}$, dont il étudie les règles de calcul ${ }^{35}$.

C'est cette méthode que les algébristes anglais reprennent et développent. Ainsi, D. H. Gregory, en 1839 , utilise ce principe pour résoudre de façon systématique certaines équations différentielles. Il note, à la fin de son article ${ }^{36}$ :

Nous avons fait comme si il y avait une distinction entre ce qui est usuellement appelé symboles d'opération, et ce qui est appelé symboles de quantité. Mais nous pourrions de façon parfaitement appropriée appeler ces derniers également symboles d'opération. Par exemple, $x$ est l'opération désignée par $(x)$ effectuée sur l'unité, $x^{n}$ est la même opération effectuée $n$ fois successivement sur l'unité (...). Si donc, nous adoptons ce point de vue (..), nous n'aurons aucune difficulté à percevoir la correction des principes par lesquels d'autres opérations, telles que celles que nous représentons par $\left(\frac{d u}{d x}\right),(\nabla)$, etc, sont traitées de la même manière que $a$, $b$, etc. Car tout ce qui est prouvé de ces derniers symboles, à partir des lois connues de leurs combinaisons, doit être également vraie de tous les autres symboles qui sont sujets aux mêmes lois de combinaison.

${ }^{35}$ Sur Arbogast, voir Jean-Pierre Friedelmeyer, Le calcul des dérivations d'Arbogast dans le projet d'algébrisation de l'analyse (Paris, Blanchard, 2003).

36 Duncan F. Gregory, On the solution of linear differential equations with constant coefficients. Cambridge Mathematical Journal, I/25-36 (1839) - Cité d'après The Mathematical Writings of Duncan F. Gregory, W. Walton ed. (Cambridge, CUP, 1864), 24. 
Ce n'est pas tant la fin du passage, où une justification en termes de lois algébriques est esquissée, que l'argument rhétorique selon lequel les quantités sont elles-mêmes des opérations qui nous intéresse ici. On retrouve en effet le même propos sous la plume de A. de Morgan, par exemple, dans le chapitre 4 du livre II de Trigonometry and double algebra :

Le sujet de l'arithmétique est le nombre; sa première opération est compter ou énumérer. (...) Si $a$ et $b$ sont deux entiers, la direction que nous donnons en formant le symbole arithmétique $a+b$ est «procède de $a$, formé en premier lieu, de la même façon que tu procèdes à partir de 0 pour former $b$ ». Maintenant si $a$ et $b$ représentent des nombres, nous devons bien entendu penser au nombre lorsque nous faisons cela. Néanmoins la description de l'opération ne contient aucune idée numérique (...) C'est seulement « Fais avec $X$ comme tu as fait avec $Y$ pour faire $Z$ », et tout livre d'art [every book of art], quel que soit son sujet, abonde de ces sortes d'instruction. Dans notre algèbre symbolique, cela se montre ainsi : $B$ est $0+B$, de sorte que, dans $A+B$, on voit que $A$ prend simplement la place de $0 .{ }^{37}$

Une quantité quelconque $B$ peut être définie tout autant comme un objet $B$ que comme une opération $+B-1$ 'opération qui, par exemple, appliquée à $A$ donne $C=A+B$. En termes à peine modernisés (puisque ce seront bientôt ceux de Cayley), on pourrait dire qu'au lieu de considérer seulement le groupe additif des entiers relatifs, de Morgan, comme Gregory, lui substituent constamment le groupe de transformations associé qui agit sur lui. Les deux mathématiciens passent ainsi continuellement des objets aux opérations et des opérations aux objets. Et ce passage est une conséquence directe de leur adhésion au « principe de séparation des symboles ».

\footnotetext{
${ }^{37}$ De Morgan, Trigonometry..., op. cit. in note 23, 115
} 
Il semble que l'analyse que A. de Morgan effectue de la notion de composition de rapport doit être replacée dans ce contexte large. Lorsqu'il présente ce concept, de Morgan, on l'a vu, note qu'il faut accomplir un pas supplémentaire dans l'abstraction pour considérer les rapports, c'est-à-dire les relations entre grandeurs, comme des grandeurs. Ce pas supplémentaire correspond précisément à celui qui consiste à traiter une opération sur les grandeurs comme une quantité. Certes, ce n'est pas la terminologie de l'opération, mais celle de la relation qui est convoquée ; et ce point est important, j'y reviendrai. Mais l'essentiel est là : saisir ce qu'est la composition de rapports, exige que l'on puisse passer sans difficulté du point de vue de la quantité à celui de l'opération sur (de la relation entre) des quantités

Citons un passage particulièrement intéressant de The Connection of Number and

\section{Magnitude :}

Lorsque nous disons deux, nous faisons référence à des répétitions (du plus petit terme) dans le rapport entre des grandeurs, reliées de cette manière :

Lorsque nous disons double de deux [twice two], nous changeons d'idiome. Au lieu de dire que le double de deux est quatre, nous pourrions dire deux deux font quatre; c'est-à-dire, là où existe l'idée d'une grandeur relative que nous exprimons par deux, nous associerons à l'idée de relation l'idée de relation plus grande exactement comme (nous associons à) l'idée de grandeur que nous avons lorsque nous regardons ---- (à celle que nous avons) lorsque nous regardons ---- ----; et nous serons conduit, en 
considérant le résultat comme un résultat de grandeur relative, à l'idée de la relation entre ---- ---- ---- ---- et ----. ${ }^{38}$

Le double désigne ici l'opération qu'il faut faire subir à ---- pour le transformer en ---- ---- ; or, cette opération, explique de Morgan, peut être elle-même séparée abstraitement de son point d'application (le segment ----) et traitée comme une grandeur, le deux, susceptible d'avoir un rapport avec d'autres grandeurs; ainsi, appliquée au résultat de sa première application, la grandeur-opération « deux » donne ---- ---- ---- ----. Tout ceci nous conduit naturellement à voir dans cette opération l'engendrement d'une nouvelle opération ou relation, celle qu'a --- ---- ---- --- avec ----. La composition des rapports paraît donc ici être conçu comme un exemple d'application du «principe de séparation des symboles » : au lieu de considérer une grandeur $B$ dans un rapport donnée $(\alpha)$ à une grandeur $A$ initiale, puis de considérer la nouvelle grandeur $C$ dans un rapport donné $(\beta)$ à la grandeur $B$, on calcule, « compose », directement les rapport $(\alpha)$ et $(\beta)$, qui appliqués à $A$ donne la grandeur $C$. On n'a pas seulement : $B=(\alpha) A$ et $C=(\beta) B$, ou $C=(\beta)[(\alpha) A]$; mais on a : $C=[(\beta)(\alpha)] A$. Dans la dernière expression, les rapports, comme s'ils étaient des quantités, sont directement composés.

Mon hypothèse est donc la suivante. A. de Morgan présente une théorie, classique, de la composition des rapports : il prend pour point de départ l'analogie entre rapports et grandeurs, et construit une addition des ratios sur le modèle de l'addition des grandeurs. La possibilité de trouver des intermédiaires (des termes médians) joue, comme dans les approches traditionnelles, le rôle décisif. L'intéressant est que cette présentation est insérée dans le contexte post-lagrangien des mathématiques de l'école algébrique anglaise. Le principe de la séparation des symboles constitue le cadre à partir duquel la doctrine

\footnotetext{
${ }^{38}$ De Morgan, The Connection..., op. cit. in note 2, 65.
} 
traditionnelle de la composition des rapports est appréhendée : le fait de pouvoir penser les grandeurs comme des opérations ou des relations conduit naturellement à effectuer l'abstraction supplémentaire demandée, et concevoir les relations entre grandeurs comme des grandeurs.

La façon dont A. de Morgan justifiait l'introduction de sa théorie des échelles relatives était articulée à sa distinction entre calcul et interprétation. Sa lecture de la doctrine de la composition des rapports pointe vers un autre contexte, celui du calcul des opérations ${ }^{39}$. Dans son traité, A. de Morgan semble donc considérer Euclide comme un contemporain, un collègue proche, qui adopte vis-à-vis de son œuvre mathématique à peu près les mêmes conceptions que les siennes. Il est surprenant de constater que, encore au milieu du XIX ${ }^{\text {ème }}$ siècle, des pratiques mathématiques se diffractent et se reflètent dans les lectures que les mathématiciens font des livres d'Euclide.

\section{III- Syllogisme et théorie de la composition.}

Revenons à notre interrogation initiale : y a-t-il une relation entre l'analyse du concept de rapport et la théorie logique des relations que développe par ailleurs le mathématicien? Il semble qu'il faille répondre positivement à cette question. L'auteur affirme en effet explicitement qu'il existe une analogie entre la théorie de la composition et sa théorie relationnelle du syllogisme, analogie qu'il juge d'ailleurs suffisamment forte pour devoir

\footnotetext{
${ }^{39}$ Il semble d'ailleurs que cette idée d'un calcul des opérations joue également un rôle dans la définition des échelles relatives. La «quantuplicité » d'une grandeur, c'est-à-dire à l'ensemble des multiples d'une même grandeur $A$, n'est en effet rien d'autre que l'orbite de 0 par l'opération $+A$. L'idée qu'une grandeur est aussi une opération se retrouve ainsi au coeur de la représentation des rapports en termes d'échelle.
} 
préciser que sa conception du syllogisme n'est pas une simple extension analogique de la théorie euclidienne de la composition.

Avant d'entrer dans le détail, soulignons un point important. Si l'arrière-plan de la théorie de la composition des rapports est fourni par le calcul des opérations, il est remarquable que de Morgan ne parle jamais, dans ce contexte, d'opérations, mais bien toujours de relations. Si les rapports avaient été considérés comme des opérations, et la composition de rapports comme une composition d'opérations, le lien à l'algèbre aurait été immédiat - mais celui que A. de Morgan établit plus tardivement avec la logique, impossible. Le poids de la tradition (de la définition V, 2 d'Euclide notamment) explique certainement pourquoi le mathématicien préfère parler de relation. Ce qui importe n'est cependant pas tant les raisons d'un tel emploi que ses conséquences : cet usage dégage tout un champ d'analyse (l'analogie entre théorie des rapports et syllogisme) que le terme d'opération aurait recouvert.

A de Morgan développe ses recherches logiques à partir de la fin des années 1840. Sa doctrine reste complètement aristotélicienne en ses fondements. Il ne s'agit jamais, pour le mathématicien, de bouleverser la structure du syllogisme, considéré comme un modèle indépassable, mais seulement de réinterpréter ses concepts fondamentaux. Une des grandes idées de A. de Morgan est notamment que la copule aristotélicienne doit être généralisée. En quoi consiste cette généralisation? L'auteur estime que deux copules sont usuellement utilisées : le « est » des logiciens, et le «=» des algébristes. Caractérisant ces copules par les propriétés qui sont nécessaires à l'effectuation d'une déduction syllogistique, il affirme, dans un article ${ }^{40}$ daté de 1850 :

${ }^{40}$ On the Symbols of Logic, the Theory of the Syllogism, and in particular of the Copula, Trans. Camb. Phil. Soc., IX, 1850, 79-127, cité d'après le recueil A. de Morgan - On the Syllogism and Other Logical Writings, P. Heath éd. (Londres, Routledge, 1966), 22-66. A. de 
Si une copule abstraite avait été utilisée, les conditions de la copularité [the copular conditions] auraient été posées de façon explicite. Il y en a deux, ensembles suffisantes pour toutes les formes d'inférence, mais non nécessaires toutes les deux. La première est ce que j'appelle la transitivité [transitiveness] (...). La seconde est la convertibilité, (...) dans laquelle la relation est sa propre corrélation. ${ }^{41}$

Autrement dit, ce n'est pas tant le contenu de la copule « est » ou «=» qui importe dans le déroulement d'une inférence que la présence d'une relation entre le sujet et le prédicat qui possède les propriétés de transitivité et de symétrie.

Cette idée conduit l'auteur à se demander ce qui se passe lorsqu'une des conditions (ou les deux) est levée. Concentrons-nous sur la condition de transitivité, qui illustre très bien la démarche de A. de Morgan. Peut-il y avoir une théorie du syllogisme qui use d'une copule non transitive? Oui, répond le mathématicien, à condition d'élaborer une théorie « bicopulaire » du syllogisme ${ }^{42}$ :

Morgan y caractérise son approche de la copule ainsi (Ibid., 50) : « Dans mes travaux en Logique Formelle (...) j'ai suivi la suggestion donnée par l'algèbre, et séparé la forme essentielle des caractéristiques accidentelles de la copule, montrant par là les conditions de l'invention d'une copule différente de la copule ordinaire, ou d'une copule qui, étant substituée à celle ordinaire, laisserait toutes les formes et les conditions de l'inférence inchangées. »

${ }^{41}$ Ibid, 51.

${ }^{42}$ Ibid., 55. 
On a insisté, dans toutes les sortes de logique, qu'elle soit formelle ou appliquée, pour avoir une copule transitive. Ceci n'est cependant pas nécessaire : une inférence peut être obtenue sans elle. La perception de relations par le biais de relations ne nécessite pas d'employer une seule relation. Si je peux voir que :

Tout $X$ a une relation à certains $Y$

Tout $Y$ a une relation à certains $Z$,

il suit que tout $X$ a une relation composée à certains $Z$. Quelles que soient les relations prémisses, il y a une relation conclusion, qui peut ou non être exprimée par un mot. Ainsi si John peut persuader Thomas, et Thomas peut commander William, nous ne pouvons pas inférer que John peut soit persuader soit commander William ; mais si nous exprimons par un mot le processus de parvenir à ses fins en persuadant celui qui peut commander - nous choisirons d'employer le mot contrôler - alors John peut contrôler William. Nous avons donc un syllogisme bicopulaire, dans lequel l'intransitivité des copules individuelles est compensée par l'invention d'une copule composée dans la conclusion. C'est la voie par laquelle nous accédons à la théorie générale de la copule.

Selon A. de Morgan, il faut concevoir la copule comme une relation, et le syllogisme, tout du moins le syllogisme de première figure (je reviendrai sur ce point), comme une composition de deux relations (Russell nommera cette composition, un produit relationnel). Si la relation est transitive, alors la relation obtenue dans la conclusion sera, évidemment, la même que les relations utilisées dans les prémisses ; mais si ce n'est pas le cas, le syllogisme est toujours 
possible : il y aura une relation entre le terme mineur et le terme majeur dans la conclusion, même si cette relation composée ne se réduit à aucune des deux relation-copules utilisées dans les prémisses. La transitivité de la copule n'est donc pas une condition sine qua non du syllogisme ; comme le dit de Morgan, « la perception de relations par le biais de relations ne nécessite pas d'employer une seule relation ».

La question de la transitivité de la copule manifeste clairement la conception que A. de Morgan se fait du syllogisme de première figure. On peut, en mettant de coté tout ce qui relève de la quantité, schématiser la forme d'un syllogisme de première figure ainsi, $t$ étant le terme mineur, $T$ le terme majeur, $M$ le moyen terme, $R$ et $S$ des copules :

$t R M$

MST

$$
t(R . S) T
$$

où R.S est la relation composée des deux relations initiales. Le syllogisme compose une nouvelle copule, ou une nouvelle relation, à partir des deux données dans les prémisses. La restriction au cas de la première figure est importante, car le moyen terme dans cette situation, joue le rôle du terme intermédiaire entre les deux relations. Rappelons, pour expliquer ce point, ce qui se passait dans la théorie des rapports de rapports. Pour composer $A: B$ avec $C$ : $D$, il fallait trouver un terme commun $X$ tel que $A: B:: Y: X$ et $C: D:: X: Z$. Le terme commun $X$ devait apparaître comme conséquent du premier rapport, et antécédent du second. Dans les syllogismes de première figure, le moyen terme joue le rôle que le terme médian a dans la composition des rapports. Ce n'est pas le cas dans les autres figures. Et A. de Morgan 
développe une théorie de la «résolution» des rapports pour rendre compte des autres configurations. L'idée, en bref, est de dire que dans tous ces autres cas, une des prémisses contient la copule complexe, et que le syllogisme extrait de la copule complexe l'autre copule. D'une certaine façon, la résolution logique est l'analogue de la recherche d'un rapport qui, composé avec un autre donné, engendre un rapport lui aussi donné.

À cause de la terminologie relationnelle employée en logique comme en géométrie (le rapport est une relation entre deux grandeurs), l'analogie entre syllogisme et théorie de la composition vient naturellement. Il n'est donc pas étonnant que A. de Morgan effectue luimême la comparaison dans un texte ultérieur, datant de 1860, On the Syllogism and on the Logic of Relations ${ }^{43}$ :

La loi suprême du syllogisme de trois termes, la loi qui gouverne tous les cas possibles, et à partir de laquelle toutes les diverses expressions doivent être considérées avant qu'une inférence puisse être faite est la suivante - une relation quelconque de $X$ à $Y$ composée d'une relation quelconque de $Y$ à $\mathrm{Z}$ donne une relation de $X$ à $Z$. Ceci constitue presque la formulation euclidienne de la définition de la composition des rapports de grandeurs - le rapport de $X$ à $Z$ est composé des rapports de $X$ à $Y$ et de $Y$ à $Z$. Si maintenant j'énonçais ce principe pour la première fois de cette manière là, on m'aurait probablement reproché d'avoir extrait une définition fantasque du syllogisme d'une analogie mathématique.

${ }^{43}$ On the Syllogism and on the Logic of Relations, Trans. Camb. Phil. Soc., X (1860) 331358, cité d'après le recueil A. de Morgan - On the Syllogism ..., op. cit. in note 40. 
Non seulement de Morgan fait explicitement la comparaison avec la théorie de la composition des rapports, mais il reconnaît que cette analogie est si forte que l'on pourrait à bon droit l'accuser de «plaquer» sur la théorie aristotélicienne un schéma de pensée d'origine euclidienne. Et de Morgan de préciser immédiatement que si cette analogie s'impose, c'est naturellement qu'elle le fait - qu'il n'a absolument pas forcé les choses, puisque c'est à la fin de son étude du syllogisme, menée complètement indépendamment de sa lecture du livre V des Eléments, qu'il la découvre :

Mais mon second article (i.e. celui de 1850, op. cit. in note 40) témoigne que j'ai énoncé l'identité entre inférence et combinaison de relations en un temps où je n'avais pas encore remarqué la très grande pertinence de l'analogie.

A. de Morgan précise toutefois dans une note attenant à l'extrait précédent, qu'il ne rejette absolument pas, bien au contraire, l'analogie :

Mais, aurais-je généralisé la notion mathématique à partir des grecs, le processus n'en aurait pas perdu pour autant son caractère naturel et sa validité. Car « rapport » n'est pas la traduction directe de logos : le mot grec signifie communication; et la même idée, qui permet de faire du logos un terme technique de la géométrie, permet de rapporter le mot, en un de ses sens dérivés, à une relation quelconque, ou encore, à n'importe quelle manière dont on parle d'une notion en termes d'une autre. N'importe quelle façon de parler d'une notion en relation à une seconde, combinée à n'importe quelle façon dont on parle de la seconde en relation à une troisième, doit dicter une manière de parler de la première en relation à la troisième. Et c'est cela, le syllogisme : 
il exhibe, dans sa forme la plus générale, la loi de la pensée qui connecte deux notions à l'aide de leur connexion à une même troisième.

Si le début de l'extrait n'est pas très convaincant (A. de Morgan y affirme que quand les grecs parlent de ratio, il parle en fait du discours et de son enchaînement), la fin, elle, est forte : dans le syllogisme comme dans la composition des rapports, on cherche à exhiber la loi qui « connecte deux notions à l'aide de leur connexion à une même troisième ». Dans le syllogisme comme dans la théorie des rapports, une même logique sous-jacente se dévoile : la logique des relations et de la composition des relations. C'est précisément la présence silencieuse de cette structure relationnelle qui fonde, selon l'auteur, la légitimité de l'analogie. On voit pourquoi il est crucial d'insister, comme je l'ai fait au début de la section, sur le fait que de Morgan parle de relations et non d'opérations dans sa théorie des rapports. S'il avait conçu les rapports comme des transformations d'une grandeur en une autre, il aurait été beaucoup plus difficile de lier les Eléments aux Seconds Analytiques, car une copule ne peut pas être considérée comme une opération qui transforme un sujet en un prédicat. En revanche, voir dans la copule une relation, un lien, est une évidence que nul ne songe à nier - et par cette voie, l'importation d'un thème géométrique au coeur de la logique classique devient possible.

Dernier point avant de conclure. Les travaux de A. de Morgan en logique n'ont pas une portée didactique - ils sont polémiques. De Morgan cherche avant tout à critiquer la doctrine dite de la «quantification du prédicat» de Hamilton. Rester fidèle aux intentions d'Aristote n'est donc pas non plus ici la préoccupation principale du mathématicien. Pourtant, les interprétations suggérées par de Morgan ne sont pas, quand on les considère comme des hypothèses historiques, complètement dénuées d'intérêt. Ainsi, la question de savoir pourquoi Aristote privilégie les formes de première figure est une des interrogations centrales de la 
littérature secondaire. Or, la réponse avancée par de Morgan, celle consistant à concevoir le syllogisme comme une composition de relations, a été encore récemment reprises par des commentateurs ${ }^{44}$.

\section{Conclusion}

J'ai cherché ici à montrer que la lecture que A. de Morgan fait du livre V d'Euclide doit être insérée dans le contexte plus vaste de sa propre pratique mathématique. Plus précisément, nous avons vu que la notion d'échelle relative était conçu par le mathématicien comme une représentation intuitive permettant d'interpréter la définition formelle que donnait Euclide, exactement comme l'interprétation géométrique des complexes donne un contenu au nombres imaginaires manipulés par les algébristes du XVII ${ }^{\text {ème }}$ et du XVIII ${ }^{\text {ème }}$. Nous avons, en second lieu, analysé comment la doctrine de la composition des rapports, telle qu'elle était présentée par A. de Morgan, s'insérait parfaitement naturellement dans le cadre de la théorie de la séparation des symboles. Enfin, j'ai montré comment cette même doctrine faisait l'objet d'une seconde lecture, non algébrique mais logique, dans laquelle la composition des rapports était perçue comme une instance d'un principe général gouvernant les syllogismes parfaits.

Le propos n'était pas de montrer que les interprétations de A. de Morgan étaient originales ou historiquement pertinentes. Il était, au contraire, de souligner que la façon dont A. de Morgan interprétait les Eléments se greffait sur des thématiques spécifiques, développées dans les mathématiques anglaises du XIX ${ }^{\text {ème }}$ siècle.

\footnotetext{
${ }^{44}$ Gunther Patzig, Die aristotelische Syllogistik, (Göttingen, Vandenhoeck \& Ruprecht, 1969) ; T. Ebert, Les syllogismes parfaits, Kaïros, 9 (1997) 76-97. Aucun des deux ne cite A. de Morgan.
} 
J'ai évoqué dans l'introduction la discussion suscitée par les interprétations « logifiantes » de Gardiès, et posé la question d'un possible lien entre la genèse de la théorie logique des relations dans la seconde moitié du $\mathrm{XIX}^{\text {̀̀me }}$ siècle, et la réception de la théorie des rapports euclidiens. Avons-nous avancé dans cette voie?

Il semblerait qu'un lien existe bel et bien entre la doctrine de la composition des rapports et la théorie relationnelle de la copule. Russell approfondira ce qui est esquissé chez A. de Morgan. Dans la sixième partie des Principia, on trouve une généralisation de la théorie des rapports, exposée dans le cadre de la nouvelle logique. Brièvement dit, Russell met en place une structure, qu'il appelle famille de vecteurs, dont les éléments (les vecteurs, qui sont des relations) correspondent aux grandeurs. À partir de là, Russell définit explicitement les rapports comme des relations de relations, et une théorie de la composition des rapports trouve dans ce contexte son cadre naturel. Les familles de vecteur sont une structure très faible -- non closes par composition, ni ordonnées (donc ni continues ni archimédiennes, ni infinies, ...). En posant des conditions de plus en plus restrictives sur ces structures, Russell et Whitehead parviennent à retrouver les propriétés familières des grandeurs euclidiennes ${ }^{45}$. Même chez celui qui est considéré comme le père de la nouvelle logique, les notions de relation et de produit relationnel sont ainsi liées à une analyse des concepts de rapports et des grandeurs.

Bien entendu, il y a loin entre dire que la logique des relations a une histoire dans lequel le livre V d'Euclide a joué un rôle important, et prétendre, comme le fait Gardiès, que l'on peut asseoir sur des distinctions logiques des hypothèses concernant la genèse du texte euclidien. Mais le propos n'était pas ici de prendre parti, pour ou contre, la lecture proposée

\footnotetext{
${ }^{45}$ Voir pour plus sur ce sujet, Sébastien Gandon, Which arithmeticisation for which logicism ? Russell on relations and quantities in The Principles of Mathematics, History and
} Philosophy of Logic. 29/1 (2008), 1-30. 
par le philosophe nantais. L'objectif était simplement d'attirer l'attention sur le fait qu'opposer frontalement les lectures historicistes aux lectures logicistes d'Euclide pourrait conduire à masquer que les théories logiques, en leur émergence même, ont eu partie liée avec la théorie des proportions. En bref, ce que j'ai tenté de suggérer ici était que l'opposition entre interprétation « logicienne » et « historiciste » devait être évaluée aussi à l'aune de l'histoire de la logique, et à celle de l'histoire de la réception, encore très riche, du texte euclidien au $\mathrm{XIX}^{\mathrm{ème}}$ siècle 